\title{
Single-nucleus RNA-sequencing in pre-cellularization Drosophila melanogaster embryos
}

\author{
Ashley Albright ${ }^{1}$, Michael Stadler ${ }^{1}$, Michael Eisen ${ }^{1,2}$ \\ ${ }^{1}$ Department of Molecular and Cell Biology, University of California Berkeley, United States \\ ${ }^{2}$ Howard Hughes Medical Institute, United States
}

\begin{abstract}
Our current understanding of the regulation of gene expression in the early Drosophila melanogaster embryo comes from observations of a few genes at a time, as with in situ hybridizations, or observation of gene expression levels without regards to patterning, as with RNA-sequencing. Single-nucleus RNA-sequencing however, has the potential to provide new insights into the regulation of gene expression for many genes at once while simultaneously retaining information regarding the position of each nucleus prior to dissociation based on patterned gene expression. In order to establish the practicality of single-nucleus RNA sequencing in the context of a real biological question, here we look at the difference in gene expression between control and an insulator protein, dCTCF, maternal null embryos during zygotic genome activation at nuclear cycle 14 . We find that early embryonic nuclei can be grouped into distinct clusters according to gene expression. From both virtual and published in situ hybridizations, we also find that these clusters correspond to spatial regions of the embryo. Lastly, we present multiple examples of differential gene expression between control and maternal CTCF null nuclei in one or more clusters, but not in bulk when grouping expression across all nuclei. These results highlight the potential for single-nucleus RNA-sequencing to reveal new insights into the regulation of gene expression in the early Drosophila melanogaster embryo.
\end{abstract}

\section{Introduction}

Early animal development is largely driven by maternally-deposited RNAs and proteins. In Drosophila melanogaster, zygotic gene expression is detected as early as the 10th nuclear cycle; however, zygotic genome activation primarily occurs during the $14^{\text {th }}$ nuclear cycle while maternal RNAs are degraded and cellularization begins[1,2]. Much of the difficulty in understanding the regulation of early embryonic gene expression lies in our ability to simultaneously capture expression level and patterning. Classic examples of patterned gene expression and regulation originate from in situ hybridizations[3-6], however the nature of in situ hybridizations does not allow for the study of many genes at once. In order to fully understand the regulation of gene expression across the genome, it is imperative that we establish new methods to examine changes in spatially-patterned genes.

Prior work from our lab demonstrated the use of RNA sequencing in patterning mutants following cryosectioning embryos across the anterior-posterior axis[7]. This work benefits from knowledge of the origin of each slice during analysis; however, with this method we cannot truly resolve from where the RNAs originated as many nuclei will contribute to expression within each slice. Recent work from Karaiskos, Whale, et al (2017) demonstrated the use of single-cell RNA-sequencing in the early Drosophila embryo and the ability to construct virtual in situ hybridizations from prior knowledge of patterned gene expression[8]. Others have used 
single-cell RNA-sequencing in dorsoventral mutant embryos and showed depletion of an entire subset of cells[9]. These studies demonstrate the potential for single-cell RNA-sequencing to answer questions relating to pattern and body axis formation in the early Drosophila embryo; however, cellular measurements may not necessarily reflect zygotic gene expression due to the presence of maternal and cytoplasmic RNAs in cells. Additionally, to understand the establishment of gene expression prior to zygotic genome activation and cellularization, we need the ability to conduct single-nucleus RNA-sequencing instead.

To establish the use of single-nucleus RNA-sequencing in the early Drosophila embryo, we decided to examine changes in gene expression in maternal null dCTCF embryonic nuclei, subsequently referred to as $d C T C F^{\text {mat- }-}$. Insulator elements were first described for their enhancer-blocking activity[10-12], and have since been shown to affect genome and chromosome structure as well[13-18]. Interestingly, mammalian CTCF serves as the only insulator protein in mammals; however, Drosophila and other arthropods have evolved several insulator proteins[19,20]. The redundancy of Drosophila insulator proteins allows us to understand the many functions of insulators without causing cell lethality. Intriguingly however, $\mathrm{dCTCF}$ is not actually required for embryonic viability[21]. Previous reports indicate that loss of individual Drosophila insulator proteins yields minimal changes in gene expression[19,22-25], but others show that $\mathrm{dCTCF}$ is required for correct expression of certain genes observed by in situ hybridizations in embryos and larvae[26,27]. The observed changes are slight however, which may explain why similar observations have not been found via sequencing.

Using 10x Genomics, we assayed gene expression across over 8,000 nuclei from control and $d C T C F^{\text {mat- }-}$ embryos. Overall, the nuclei tend to cluster according to expression of

spatially-patterned genes, indicating that the nuclei retain information regarding their position in the embryo prior to dissociation. This allows us to understand differential expression in spatial regions by sequencing, which was previously only possible by mechanical manipulation. As expected considering the viability of $d C T C F^{\text {mat- }-}$ embryos, we found fewer differentially expressed genes in bulk than in individual clusters. We also found several examples of patterned genes that are differentially expressed in certain clusters but not in bulk. Our analyses are available in a reproducible and usable format (see Code Availability) allowing others to explore our data analysis as well as analyze other genes of interest not explored here. Altogether this work encompasses a resource to explore the effect of dCTCF on gene expression, as well as establishes the use of single-nucleus RNA-sequencing in the early Drosophila embryo.

\section{Methods}

\section{CRISPR}

CTCF nulls were created by using CRISPR mutagenesis to insert a dsRed protein followed by two consecutive stop codons immediately upstream of the CTCF open reading frame. The homologous replacement template plasmid was constructed using a pUC19 backbone and $\sim 1 \mathrm{~kb}$ homology arms generated by PCR ( 5 ' homology arm primers: CCACAAAGAAACGTTAGCTAGTTCC and TCCTATGGACAAATTGGATTTGTTTTGG, 3' homology arm primers: CCAAGGAGGACAAAAAAGGACGAG and CGTGAGTGGCGCGTGATC). Repair template was coinjected into Cas9-expressing embryos 
(Rainbow Transgenic Flies, Camarillo, California), along with two guide RNAs (ATTTGTCCATAGGAATGCCA, TGTCCATAGGAATGCCAAGG) expressed from a U6:3 promoter on a modified version of the pCFD3 plasmid[28]). Resulting flies were crossed to flies containing chromosome 3 balancer chromosomes, and screened by genotyping PCR. Putative hits were further screened by PCR and sequencing of the entire locus using primers outside the homology arms (CATTAGAATTCAAGGGCCATCAG and CACTTGAAGGATGGCTCG). A successful insertion line was recombined with an FRT site on chromosome 3L at cytosite 80B1 (Bloomington stock \# BL1997).

\section{Fly husbandry}

All stocks were fed standard Bloomington food from LabExpress and maintained at room temperature unless otherwise noted. We used the FLP-DFS (dominant female sterile) technique[29] to generate $d C T C F^{\text {mat-/ }}$ embryos. First, we crossed virgin $h s F L P, w^{*} ; ; G l^{*} / T M 3$ females to $w^{*} ; ;$ ovo ${ }^{D}, F R T 2 A(m w) / T M 3$ males (Bloomington Drosophila Stock Center ID: 2139). From this cross, we selected $h s F L P, w^{*} ; ;$ ovo ${ }^{D}, F R T 2 A(\mathrm{mw}) / T M 3$ males and crossed them to virgin $C T C F^{*}, F R T 2 A / T M 3$ females. Larvae from this cross were heat-shocked on days 4, 5, and 6 for at least two hours in a water bath at $37^{\circ} \mathrm{C}$. Upon hatching, virgin $h s F L P, w^{*} /+; ; C T C F^{*}$, $F R T 2 A(m w) / C T C F^{*}, F R T 2 A(m w)$ females were placed into a small cage with their male siblings. Flies were fed every day with yeast paste (dry yeast pellets and water) spread onto apple juice agar plates. These crosses were conducted simultaneously with another insulator protein, and control embryos were collected from the $o v o^{D}$ line used to generate those germline clones (Bloomington Drosophila stock Center ID: 2149).

\section{Western blots}

Flies laid on grape-agar plates for two hours and embryos were either aged two hours at room temperature or taken directly after collection. Embryos were dechorionated with bleach, rinsed, and frozen in aliquots of $\sim 25$ embryos at $-80 \mathrm{C}$. Embryos were homogenized in $25 \mu \mathrm{l}$ RIPA buffer (Sigma cat \# R0278) supplemented with $1 \mathrm{mM}$ DTT and protease inhibitors (Sigma cat \# 4693116001) using a plastic pestle. After homogenization, samples were mixed with $25 \mu 12 \mathrm{x}$ Laemmli buffer (Bio-Rad \# 1610737EDU), boiled for 3 minutes, and spun at 21,000 x g for 1 minute. Samples were loaded onto Bio-Rad mini Protean TGX 4-20\% gels (\# 4561096) and run at $200 \mathrm{~V}$ for 30 minutes. Protein was transferred at $350 \mathrm{~mA}$ for one hour to Immobilon PVDF membrane (Millipore-Sigma \# IPVH00010). Blots were blocked for one hour in PBST (1x PBS with $0.1 \%$ Tween) with $5 \%$ nonfat milk, and stained with primary antibodies (courtesy of Maria Cristina Gambetta[27], 1:1000 in PBST with 3\% BSA) for one hour. Blots were then washed 3 times for 3 minutes rotating in PBST and probed with an HRP-conjugated anti-Rabbit secondary antibody (Rockland Trueblot, \# 18-8816-33, 1:1000 in PBST with 5\% milk) for one hour. After extensive washing with PBST, blots were developed with Clarity ECL reagents (Bio-Rad \# 1705060) and imaged. Validation of the loss of maternal dCTCF is shown in Supplementary Figure 1.

\section{Nuclear isolation and sequencing}


Nuclei were isolated from early to mid-nuclear cycle 14 embryos (stage 5) according to several previously published works[30-32]. Control nuclei were collected from embryos First, the cages were cleared for 30 minutes to 1 hour to remove embryos retained by the mothers overnight, followed by a 2 hour collection and 2 hour aging. Then, the embryos were dechorionated in $100 \%$ bleach for 1 minute, or until most of the embryos were floating, with regular agitation by a paintbrush. The embryos were transferred to a collection basket made of a $50 \mathrm{~mL}$ conical and mesh. After the embryos were rinsed with water, the embryos were transferred into an eppendorf tube containing $0.5 \%$ PBS-Tween. From this point forward, samples were kept on ice to prevent further aging of embryos.

A minimum of 9 early to mid-nuclear cycle 14 embryos were sorted using an inverted compound light microscope and transferred to a $2 \mathrm{~mL}$ dounce containing $600 \mathrm{uL}$ of lysis buffer $(10 \mathrm{mM} 10$ $\mathrm{mM}$ Tris-HCl pH 7.4, 10 mM NaCl, 3 mM MgCl2, 1\% Bovine Serum Albumin, 1\% RNase Inhibitor (Enzymatics, Part Num. Y9240L)) + 0.1\% IGEPAL. The embryos were homogenized 20 times with a loose pestle and 10 times with a tight pestle. Pestles were rinsed with $100 \mathrm{uL}$ lysis buffer $+0.1 \%$ IGEPAL after use. The resulting $800 \mathrm{uL}$ of buffer and nuclei were transferred into an eppendorf tube, filtered with a $40 \mathrm{uM}$ filter. Nuclei were pelleted by spinning for 5 minutes at $900 \mathrm{~g}$ and $4^{\circ} \mathrm{C}$, washed in $500 \mathrm{uL}$ lysis buffer (without $0.1 \%$ IGEPAL), and pelleted again before resuspending the nuclei in $20 \mathrm{uL}$ lysis buffer (without $0.1 \%$ IGEPAL). Nuclei concentration was then adjusted to $1000 \mathrm{uL}$ nuclei per $\mathrm{uL}$, then nuclei were barcoded with the 10X Chromium Single Cell 3' Gene Expression Kit (v3). Control and $d C T C F^{\text {mat- }}$ nuclei were processed on separate days, then sequenced together with the Illumina NovaSeq (SP flow cell).

\section{Data processing and analysis}

We used kallisto-bustools[33] to generate a custom reference index and generate a nucleus $\mathrm{x}$ gene matrix. The data were analyzed in both Python and $\mathrm{R}$, using primarily scVI via scvi-tools[34,35], scanpy[36], and custom scripts for analysis.

Control and $d C T C F^{\text {mat-/ }}$ nuclei were filtered separately as follows: (1) nuclei were ranked by the number of UMIs detected and nuclei ranked below the to the expected number of nuclei $(10,000)$ were removed; (2) nuclei with fewer than 200 expressed genes were removed; (3) nuclei with greater than 5\% mitochondrial expression were removed; (4) nuclei with greater than 50,000 UMI counts were removed; (5) genes expressed in fewer than 3 nuclei were removed.

Prior to batch correction, the data were subset to the 6000 most highly variable genes using scanpy's $d C T C F^{\text {mat- }}$ based on $\log 1 \mathrm{p}$ normalized expression. We ran scVI with gene_likelihood $=$ ' $\mathrm{nb}$ ' to correct for batch effects.

The nuclei were clustered using the Leiden algorithm[37] within scanpy and visualized on a 2D UMAP[38]. Prior to batch correction, nuclei were clustered on log1p normalized gene expression. After batch correction, nuclei were clustered on the latent space derived from the scVI model. Marker genes representing each cluster were found using the sc.tl.rank_genes_groups function from scanpy with the Wilcoxon signed-rank test. In situ hybridizations of representative marker genes were obtained from the Berkeley Drosophila 
Genome Project[39-41]. Colors representing Leiden clusters were projected onto a virtual embryo using novoSpaRc[42,43].

Log2 fold change and associated p-values were obtained for each gene using diffxpy (https://diffxpy.readthedocs.io/). Significantly differentially expressed genes were determined following Bonferroni correction of the p-values and filtered for adjusted p-value less than 0.05 and absolute value of $\log 2$ fold change greater than or equal to 1.5. Intersecting sets of differentially expressed genes were found and visualized with an UpSet plot[44,45], following correction of adjusted p-values for the number of comparisons (multiplied by $11 ; 10$ for the total number of clusters +1 to include bulk differential expression).

\section{Data and code availability}

Raw sequencing files have been submitted to Data Dryad and will be available here: https://doi.org/10.6078/D13D9R

All code is available here: $\underline{\text { https://github.com/aralbright/2021_AAMSME }}$

\section{Results}

To establish the use of single-nucleus RNA-sequencing for examining gene expression prior to the onset of zygotic genome activation and cellularization, we hand-sorted 10 to 20 early to mid-nuclear cycle 14 control and $d C T C F^{\text {mat- }-}$ embryos and isolated nuclei for single-nucleus RNA-sequencing using 10x Genomics 3' Gene Expression. After filtering the data for high quality nuclei and correcting for non-biological variability (Supplementary Figures 2-4), we used Leiden clustering [37] to detect distinct groups of nuclei from control and $d C T C F^{\text {mat- }}$ embryos, altogether resulting in 8,400 nuclei across 10 clusters (Figure 1a) composed of both control and $d C T C F^{\text {mat- }}$ nuclei (Figure $1 \mathrm{~b}$ ). We also removed yolk and pole cell nuclei as these nuclei are not informative for patterned gene expression; however, the fact that subsets of nuclei clustered on marker gene expression for yolk or pole cell nuclei provided us with confidence that our data accurately represent single-nucleus expression (Supplementary Figure 5). After removing groups of nuclei as indicated, the nuclei no longer cluster according to expression of yolk or pole cell markers, indicating that our data are of high quality (Supplementary Figure 6). Once we finalized the dataset, we then asked whether gene expression in the clusters determined by the Leiden algorithm are truly distinct. 


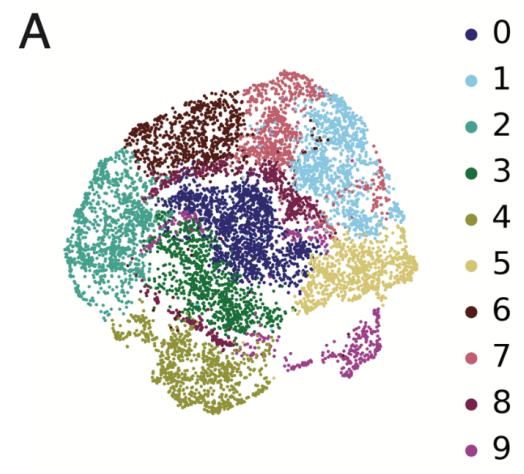

B
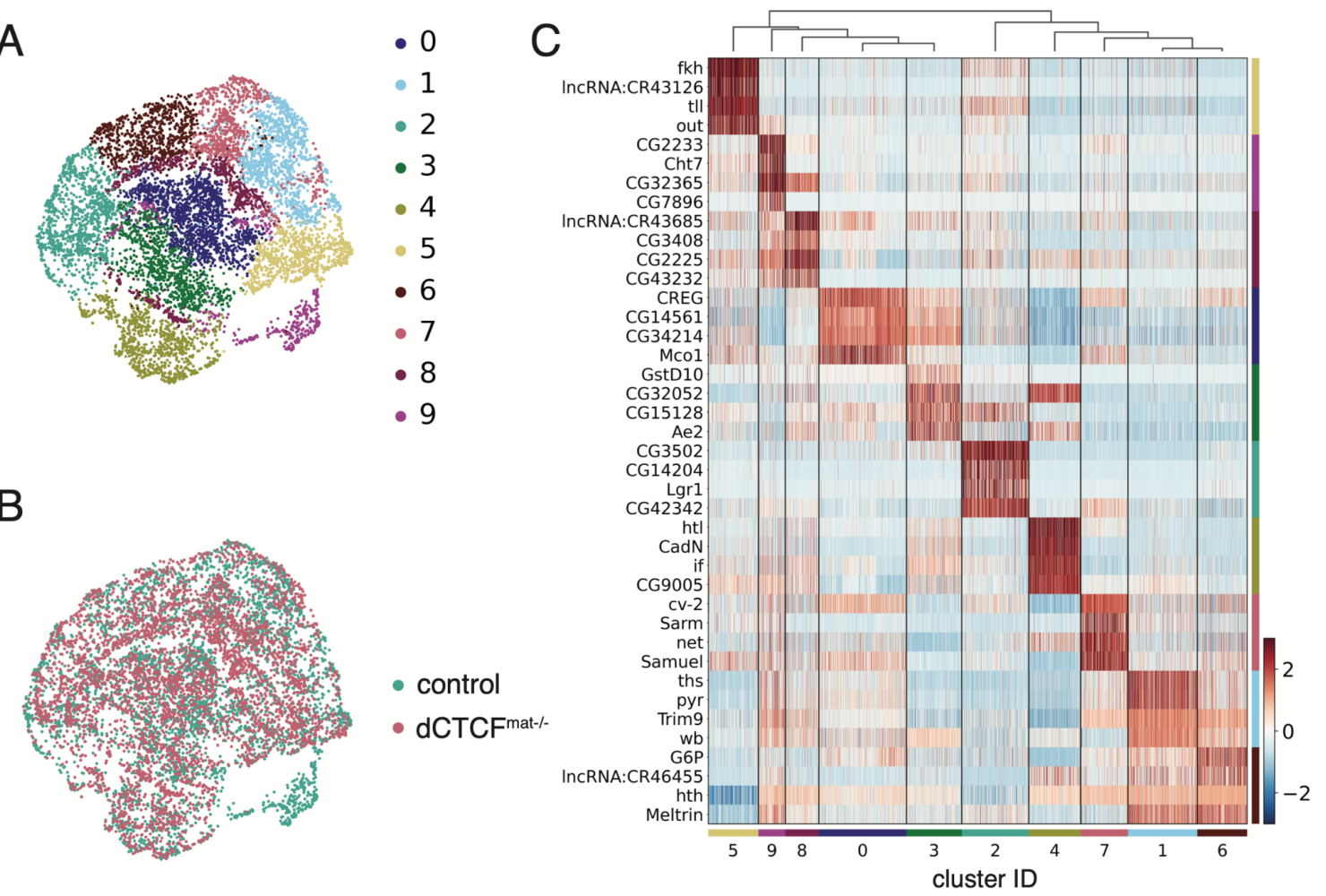

Figure 1. Single-nucleus RNA-sequencing analysis of pre-cellularization Drosophila melanogaster embryos (a) two-dimensional UMAP embedding of nuclei shows 10 transcriptionally distinct clusters, as determined using the Leiden algorithm. Associated colors are maintained throughout the manuscript. (b) two-dimensional UMAP embedding of nuclei labeled by condition shows the overlap of control and $d C T C F^{\text {mat- }}$ nuclei in a reduced dimensional space following correction for non-biological variability. Associated colors are maintained throughout the manuscript. (c) Heatmap of scaled gene expression for top four marker genes of each cluster, clusters are hierarchically ordered.

Given how well characterized patterned gene expression is in the early Drosophila embryo and that we found several distinct clusters of nuclei, we suspected that the clusters may represent different spatial regions within the embryo. Expression of the top marker genes representing each cluster is certainly distinct, and we noticed that many of these genes are expressed in patterns, namely $f k h$, $t l l$, and $h t l$ (Figure 1c). To determine if single-nucleus RNA-sequencing in the early embryo can be spatially resolved, we examined in situ hybridizations of top marker genes for each cluster. We found that representative virtual and published in situ hybridizations of the top 20 marker genes (Supplementary Table 1) correspond to specific spatial regions within the embryo for clusters 0-7 (Figure 2a-h and Figure 2a'-h'). The patterned expression patterns of marker genes did not always correspond to future tissue types or cell fates, therefore we decided to assign generic spatial regions to the nuclei instead. We also found by projecting our nuclei onto a virtual embryo that the identities we assigned to each cluster correspond to the spatial identities of these clusters (Figure 2i). 


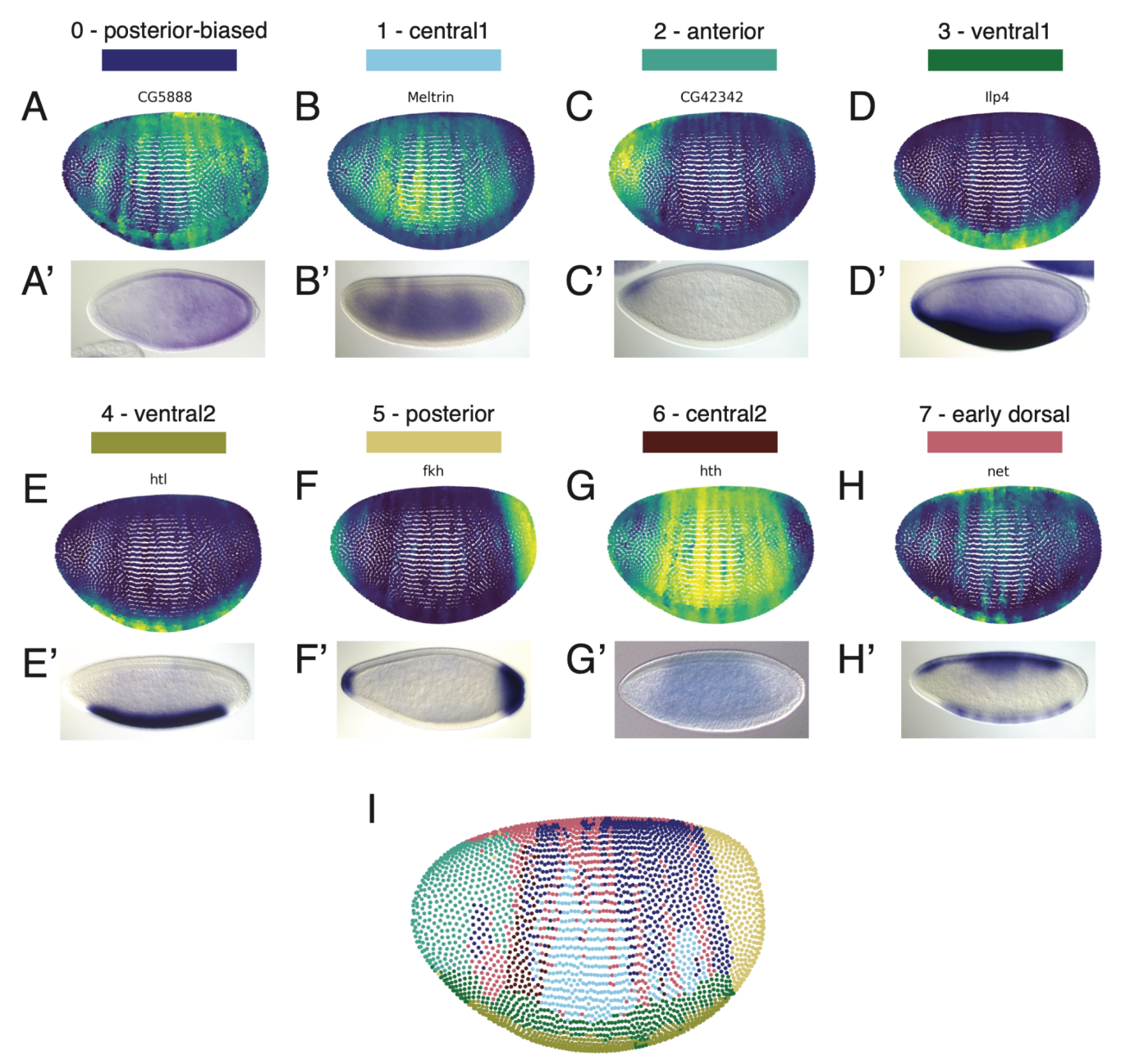

Figure 2. Leiden clusters correspond to spatial regions within the embryo (a-h)

Representative virtual in situ hybridizations (top, a-h) for top marker genes representing each cluster as labeled and the corresponding published in situ hybridizations (bottom, a'-h') from the Berkeley Drosophila Genome Project[39-41]. (i) Projection of nuclei onto a virtual embryo labeled by the Leiden cluster as colored in Figure 1a. Virtual in situ hybridizations and projection of clusters onto a virtual embryo were generated using novoSpaRc[42,43].

The anterior, posterior, and ventral clusters are the most defined based on a projection of our nuclei onto a virtual embryo, while clusters that represent the middle of the embryo in general had less well defined borders (Figure 2i). Even so, our data shows that single-nucleus RNA-sequencing in the early Drosophila embryo yields information related to the spatial position of nuclei prior to dissociation. We should note however, that the virtual in situ hybridizations shown above, as well as additional virtual in situ hybridizations that represent each cluster, contain genes present in the list of reference genes used to generate the virtual patterns (see Ilp4, htl, fkh in Figure 2, and Antp, NetA, disco in Supplementary Figure 7). As such, we considered that the virtual in situ hybridizations may be biased in those cases; however, we believe the presence of several other genes representing each cluster with similar patterning validated with both virtual and published in situ hybridizations indicates that reference bias is not 
an issue. In the end, we were unable to determine a spatial identity for clusters 8 and 9; however, we decided to include these clusters in subsequent analyses because the nuclei passed our quality control filters. In an effort to understand the effect of the loss of maternal dCTCF on zygotic gene expression, we then asked whether differential expression differs between clusters and in bulk. 
A

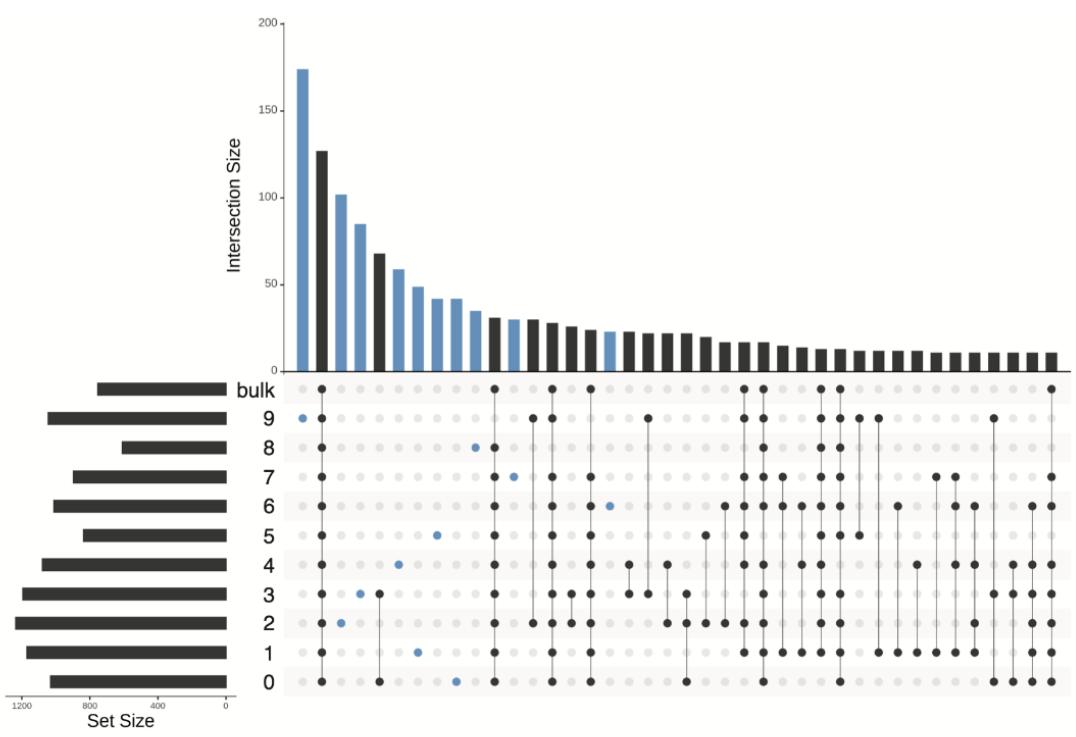

B
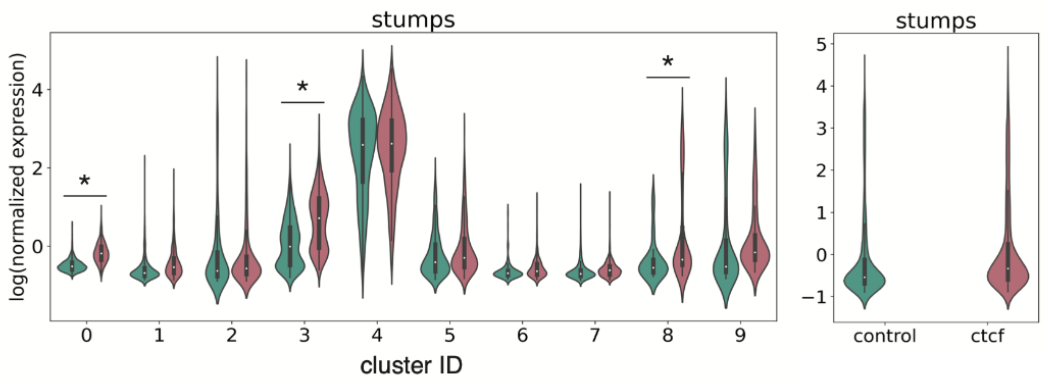

C
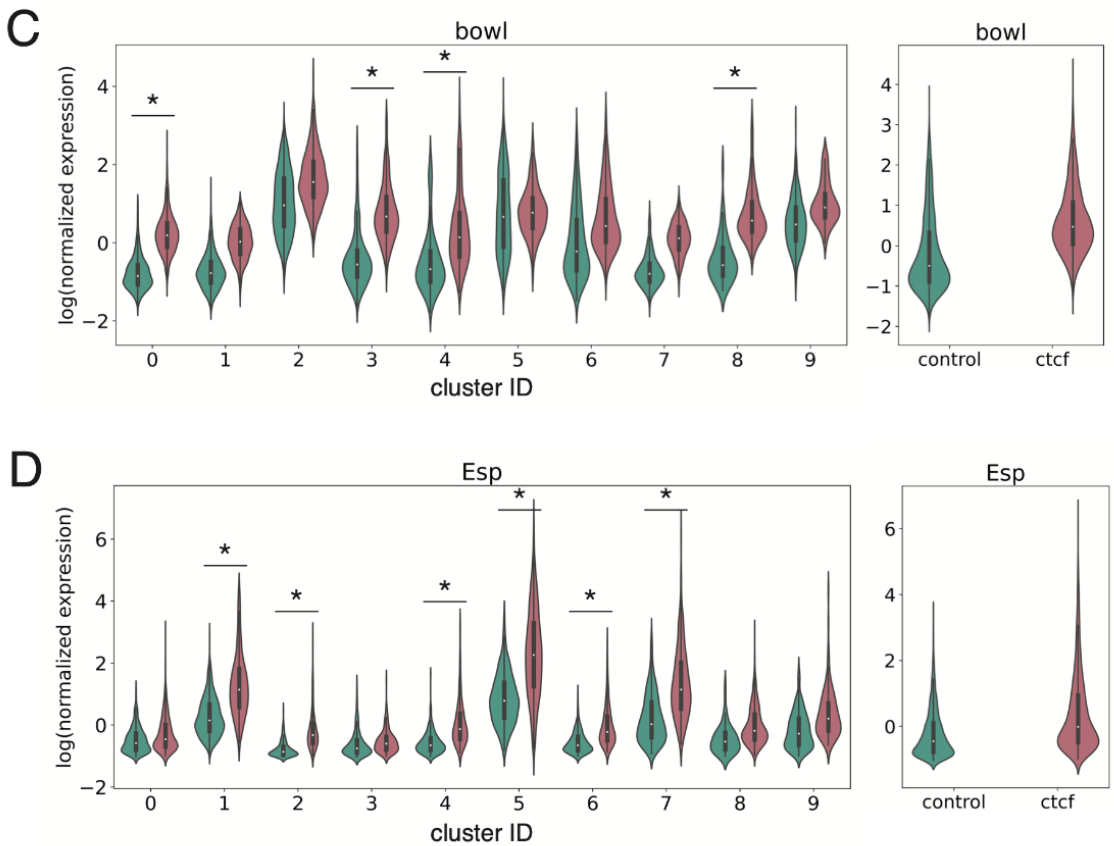

Figure 3. Differential expression of genes detected in one or more clusters, but not in bulk (a) UpSet plot for visualizing the top 40 shared sets of differentially expressed genes between control and $d C T C F^{\text {mat-/ }}$ nuclei within each cluster and in bulk. Horizontal bar plot (a, left) 
represents the total number of differentially expressed genes within the corresponding row. The vertical bar plot (a, top) represents the number of shared differentially expressed genes for the conditions indicated below and is sorted from largest to smallest intersecting set. Connected dots (black) represent the corresponding group of genes in the vertical bar plot above that are differentially expressed in the filled in circles. Genes differentially expressed in a single cluster are represented in blue. (b-d) $\log$ (scvi normalized expression) of (b) stumps, (c) bowl, (d) Esp in each cluster (left) and bulk (right) for control (teal) and $d C T C F^{\text {mat-/ }}$ nuclei (pink). Asterisks indicate significantly different expression (absolute value of expression $>=1.5$ and Bonferroni corrected p-value $<0.05$ ).

In most clusters and in bulk, gene expression tends to be up-regulated upon loss of maternal dCTCF (Supplementary Figure 8). We also found that differentially expressed genes shared between all clusters and in bulk represent one of the largest shared sets (Figure 3a, left most black bar). However, a substantial number of differentially expressed genes are differentially expressed in single clusters (Figure 3a, blue bars). Many other genes are also differentially expressed in groups of clusters, but not in bulk. Because we found many differentially expressed genes, we considered that this may be due to low expression given the sparsity of single-nucleus RNA-sequencing; however, we found that the mean expression of differentially expressed genes in single or multiple clusters overall does not have a substantially different pattern from that of non-differentially expressed genes (Supplementary Figure 9). Altogether, these results suggest that loss of maternal dCTCF may differentially impact gene expression across physical space.

Local changes in gene expression are likely lost in bulk RNA-sequencing; however, we expect to observe differential expression of patterned genes in specific clusters. Interestingly, stumps, a ventrally-expressed gene is differentially expressed in one ventral cluster, but not the other (Figure 3b). bowl, a gap gene primarily expressed in the anterior, appears to be upregulated in the posterior-biased and one of the ventral clusters (Figure 3c). Finally, Esp, a posterior-striped gene is differentially expressed in several clusters. Intriguingly, none of these examples are differentially expressed in bulk. We cannot be certain whether or not these and many other genes are truly differentially expressed spatially without further investigation; however, our results highlight the possibility of local changes in gene expression upon perturbation in the early embryo as revealed by single-nucleus RNA-sequencing.

\section{Discussion}

We conducted the above analyses in order to determine whether we could use single-nucleus RNA-sequencing as a means of understanding the regulation of gene expression in the early Drosophila embryo. First, we show that nuclei can be grouped into clusters represented by distinct gene expression. Then, we show that representative marker genes from the majority of the clusters recapitulate known patterns of expression. Importantly, we also describe examples of differential expression of patterning genes in individual clusters upon loss of maternal dCTCF, but not in bulk.

Prior to this work, studies towards our understanding of the regulation of patterned gene expression in a spatial context included cytoplasmic RNAs in measures of expression. We must acknowledge the caveat that we do not know the extent to which maternal RNAs enter the 
nucleus and some of our results may reflect the presence of both maternal and zygotic RNAs. Nonetheless, we believe that single-nucleus RNA-sequencing is better suited to understand changes in gene expression in pre-cellularization embryos upon the loss of important developmental factors because of the ability to resolve local changes in expression. Supporting this notion, single-cell RNA-sequencing has already shown to resolve the loss of an entire cell fate in dorsoventral mutant embryos[9].

Whether or not the changes in gene expression that we observed have implications in embryonic development related to the loss of $\mathrm{dCTCF}$ is unclear without further investigation, such as single-molecule FISH to validate the observed changes in gene expression of particular RNAs. Ultimately, using single-nucleus RNA-sequencing to examine changes in gene expression upon the loss of important developmental factors has the potential to uncover perturbation responses previously undetected by bulk RNA-sequencing.

\section{Acknowledgements}

We are grateful for Maria Cristina Gambetta and the generous sharing of Cp190 and dCTCF antibodies. Thank you to Dr. Justin Choi and the UC Berkeley Functional Genomics Laboratory as well as the Vincent Coates Sequencing Laboratory for processing our samples. We would also like to thank Sina Booeshaghi, Tara Chari, and Lior Pachter of the Pachter Lab at Caltech for their feedback and discussions on single-nucleus RNA-sequencing analysis. We thank Colleen Hannon and Marc Singleton and all members of the Eisen lab for helpful discussions and advice throughout preparation of the manuscript. AA was supported by an NIH Training Grant (T21 GM 007127) and the National Science Foundation Graduate Research Fellowship Program. MS was supported by an American Cancer Society postdoctoral fellowship (126730-PF-14-256-01-DDC). The work was also supported by a Howard Hughes Medical Institute Investigator award to ME.

\section{References}

1. De Renzis S, Elemento O, Tavazoie S, Wieschaus EF. Unmasking activation of the zygotic genome using chromosomal deletions in the Drosophila embryo. PLoS Biol. 2007;5: e117.

2. Harrison MM, Eisen MB. Transcriptional activation of the zygotic genome in Drosophila. Curr Top Dev Biol. 2015;113: 85-112.

3. Goto T, Macdonald P, Maniatis T. Early and late periodic patterns of even skipped expression are controlled by distinct regulatory elements that respond to different spatial cues. Cell. 1989;57: 413-422.

4. Harding K, Hoey T, Warrior R, Levine M. Autoregulatory and gap gene response elements of the even-skipped promoter of Drosophila. EMBO J. 1989;8: 1205-1212.

5. Small S, Kraut R, Hoey T, Warrior R, Levine M. Transcriptional regulation of a pair-rule stripe in Drosophila. Genes Dev. 1991;5: 827-839.

6. Small S, Blair A, Levine M. Regulation of even-skipped stripe 2 in the Drosophila embryo. 
The EMBO Journal. 1992. pp. 4047-4057. doi:10.1002/j.1460-2075.1992.tb05498.x

7. Combs PA, Eisen MB. Genome-wide measurement of spatial expression in patterning mutants of Drosophila melanogaster. F1000Res. 2017;6: 41.

8. Karaiskos N, Wahle P, Alles J, Boltengagen A, Ayoub S, Kipar C, et al. The Drosophila embryo at single-cell transcriptome resolution. Science. 2017;358: 194-199.

9. Ing-Simmons E, Vaid R, Bing XY, Levine M, Mannervik M, Vaquerizas JM. Independence of chromatin conformation and gene regulation during Drosophila dorsoventral patterning. Nat Genet. 2021;53: 487-499.

10. Kellum R, Schedl P. A position-effect assay for boundaries of higher order chromosomal domains. Cell. 1991;64: 941-950.

11. Kellum R, Schedl P. A group of scs elements function as domain boundaries in an enhancer-blocking assay. Mol Cell Biol. 1992;12: 2424-2431.

12. Holdridge C, Dorsett D. Repression of hsp70 heat shock gene transcription by the suppressor of hairy-wing protein of Drosophila melanogaster. Mol Cell Biol. 1991;11: 1894-1900.

13. Stadler MR, Haines JE, Eisen MB. Convergence of topological domain boundaries, insulators, and polytene interbands revealed by high-resolution mapping of chromatin contacts in the early Drosophila melanogaster embryo. Elife. 2017;6. doi:10.7554/eLife.29550

14. Roseman RR, Pirrotta V, Geyer PK. The su(Hw) protein insulates expression of the Drosophila melanogaster white gene from chromosomal position-effects. EMBO J. 1993;12: 435-442.

15. Sigrist CJ, Pirrotta V. Chromatin insulator elements block the silencing of a target gene by the Drosophila polycomb response element (PRE) but allow trans interactions between PREs on different chromosomes. Genetics. 1997;147: 209-221.

16. Mallin DR, Myung JS, Patton JS, Geyer PK. Polycomb group repression is blocked by the Drosophila suppressor of Hairy-wing [su(Hw)] insulator. Genetics. 1998;148: 331-339.

17. Recillas-Targa F, Pikaart MJ, Burgess-Beusse B, Bell AC, Litt MD, West AG, et al. Position-effect protection and enhancer blocking by the chicken $\beta$-globin insulator are separable activities. Proc Natl Acad Sci U S A. 2002;99: 6883-6888.

18. Kahn TG, Schwartz YB, Dellino GI, Pirrotta V. Polycomb complexes and the propagation of the methylation mark at the Drosophila ubx gene. J Biol Chem. 2006;281: 29064-29075.

19. Schwartz YB, Linder-Basso D, Kharchenko PV, Tolstorukov MY, Kim M, Li H-B, et al. Nature and function of insulator protein binding sites in the Drosophila genome. Genome Res. 2012;22: 2188-2198. 
20. Heger P, George R, Wiehe T. Successive gain of insulator proteins in arthropod evolution. Evolution. 2013;67: 2945-2956.

21. Rickels R, Herz H-M, Sze CC, Cao K, Morgan MA, Collings CK, et al. Histone H3K4 monomethylation catalyzed by Trr and mammalian COMPASS-like proteins at enhancers is dispensable for development and viability. Nat Genet. 2017;49: 1647-1653.

22. Van Bortle K, Ramos E, Takenaka N, Yang J, Wahi JE, Corces VG. Drosophila CTCF tandemly aligns with other insulator proteins at the borders of $\mathrm{H} 3 \mathrm{~K} 27 \mathrm{me} 3$ domains. Genome Res. 2012;22: 2176-2187.

23. Page AR, Kovacs A, Deak P, Torok T, Kiss I, Dario P, et al. Spotted-dick, a zinc-finger protein of Drosophila required for expression of Orc4 and S phase. EMBO J. 2005;24: 4304-4315.

24. Cuartero S, Fresán U, Reina O, Planet E, Espinàs ML. Ibf1 and Ibf2 are novel CP190-interacting proteins required for insulator function. EMBO J. 2014;33: 637-647.

25. Özdemir I, Gambetta MC. The Role of Insulation in Patterning Gene Expression. Genes . 2019;10. doi:10.3390/genes10100767

26. Gambetta MC, Furlong EEM. The insulator protein CTCF is required for correct Hox gene expression, but not for embryonic development in Drosophila. Genetics. 2018;210: $129-136$.

27. Kaushal A, Mohana G, Dorier J, Özdemir I, Omer A, Cousin P, et al. CTCF loss has limited effects on global genome architecture in Drosophila despite critical regulatory functions. Nat Commun. 2021;12: 1011.

28. Port F, Chen H-M, Lee T, Bullock SL. Optimized CRISPR/Cas tools for efficient germline and somatic genome engineering in Drosophila. Proc Natl Acad Sci U S A. 2014;111: E2967-76.

29. Chou TB, Perrimon N. The autosomal FLP-DFS technique for generating germline mosaics in Drosophila melanogaster. Genetics. 1996;144: 1673-1679.

30. Haines JE, Eisen MB. Patterns of chromatin accessibility along the anterior-posterior axis in the early Drosophila embryo. PLoS Genet. 2018;14: e1007367.

31. Bonn S, Zinzen RP, Perez-Gonzalez A, Riddell A, Gavin A-C, Furlong EEM. Cell type-specific chromatin immunoprecipitation from multicellular complex samples using BiTS-ChIP. Nat Protoc. 2012; 7: 978-994.

32. Cao J, Spielmann M, Qiu X, Huang X, Ibrahim DM, Hill AJ, et al. The single-cell transcriptional landscape of mammalian organogenesis. Nature. 2019;566: 496-502.

33. Melsted P, Booeshaghi AS, Liu L, Gao F, Lu L, Min KHJ, et al. Modular, efficient and constant-memory single-cell RNA-seq preprocessing. Nat Biotechnol. 2021;39: 813-818. 
34. Lopez R, Regier J, Cole MB, Jordan MI, Yosef N. Deep generative modeling for single-cell transcriptomics. Nat Methods. 2018;15: 1053-1058.

35. Gayoso A, Lopez R, Xing G, Boyeau P, Wu K, Jayasuriya M, et al. Scvi-tools: A library for deep probabilistic analysis of single-cell omics data. bioRxiv. bioRxiv; 2021.

doi:10.1101/2021.04.28.441833

36. Wolf FA, Angerer P, Theis FJ. SCANPY: large-scale single-cell gene expression data analysis. Genome Biol. 2018;19. doi:10.1186/s13059-017-1382-0

37. Traag VA, Waltman L, van Eck NJ. From Louvain to Leiden: guaranteeing well-connected communities. Sci Rep. 2019;9: 5233.

38. McInnes L, Healy J, Melville J. UMAP: Uniform Manifold Approximation and Projection for Dimension Reduction. arXiv [stat.ML]. 2018. Available: http://arxiv.org/abs/1802.03426

39. Hammonds AS, Bristow CA, Fisher WW, Weiszmann R, Wu S, Hartenstein V, et al. Spatial expression of transcription factors in Drosophila embryonic organ development. Genome Biol. 2013;14: R140.

40. Tomancak P, Berman BP, Beaton A, Weiszmann R, Kwan E, Hartenstein V, et al. Global analysis of patterns of gene expression during Drosophila embryogenesis. Genome Biol. 2007;8: R145.

41. Tomancak P, Beaton A, Weiszmann R, Kwan E, Shu S, Lewis SE, et al. Systematic determination of patterns of gene expression during Drosophila embryogenesis. Genome Biol. 2002;3: RESEARCH0088.

42. Nitzan M, Karaiskos N, Friedman N, Rajewsky N. Gene expression cartography. Nature. 2019;576: 132-137.

43. Moriel N, Senel E, Friedman N, Rajewsky N, Karaiskos N, Nitzan M. NovoSpaRc: flexible spatial reconstruction of single-cell gene expression with optimal transport. Nat Protoc. 2021. doi:10.1038/s41596-021-00573-7

44. Lex A, Gehlenborg N, Strobelt H, Vuillemot R, Pfister H. UpSet: Visualization of intersecting sets. IEEE Trans Vis Comput Graph. 2014;20: 1983-1992.

45. Conway JR, Lex A, Gehlenborg N. UpSetR: an R package for the visualization of intersecting sets and their properties. Bioinformatics. 2017;33: 2938-2940. 